\title{
Produção estacional, índice de área foliar e interceptação luminosa de cultivares de alfafa sob pastejo
}

\author{
María del Carmen Ferragine ${ }^{(1)}$, Carlos Guilherme Silveira Pedreira ${ }^{(1)}$, Lyssa Otani ${ }^{(1)}$, Felipe Tonato ${ }^{(1)}$ \\ (1)Universidade de São Paulo, Escola Superior de Agricultura Luiz de Queiroz, Dep. de Zootecnia, Av. Pádua Dias, 11, Caixa Postal 9, \\ CEP 13418-900 Piracicaba, SP. E-mail: mdcferra@hotmail.com, cgspedre@esalq.usp.br, lyssaot@yahoo.com, felipetonato@yahoo.com
}

Resumo - A alfafa (Medicago sativa L.) pastejada pode promover redução de custos de alimentação de ruminantes, mas o desempenho agronômico de cultivares sob pastejo no Brasil ainda é pouco conhecido. O objetivo deste trabalho foi avaliar o efeito do método de pastejo sobre o acúmulo de forragem (AF), índice de área foliar (IAF) e interceptação luminosa (IL) de cinco cultivares de alfafa, durante 295 dias de pastejo em Piracicaba, SP. O experimento seguiu um delineamento de blocos completos casualizados, com parcelas subdivididas (método na parcela e cultivar na subparcela). A cultivar ABT-805 foi a mais produtiva sob lotação contínua $\left(26.600 \mathrm{~kg} \mathrm{ha}^{-1}\right.$ ano $\left.^{-1}\right)$ e rotacionada $\left(18.000 \mathrm{~kg} \mathrm{ha}^{-1}\right.$ ano $\left.^{-1}\right)$, com maior IAF médio $(2,1)$ e o máximo valor médio de IL (53\%) sob lotação rotacionada. Alfagraze, apesar de ser uma das menos produtivas sob lotação rotacionada $\left(13.300 \mathrm{~kg} \mathrm{ha}^{-1}\right.$ ano $\left.^{-1}\right)$, foi semelhante em produtividade a ABT-805, sob lotação contínua (26.300 kg ha-1 ano $\left.{ }^{-1}\right)$, apresentando IL média de 47\% e IAF médio de 1,1 e 1,8 para a lotação contínua e rotacionada, respectivamente. Pioneer 5432 foi intermediária, com IL média de $49 \%$, AF de 24.300 e $15.300 \mathrm{~kg} \mathrm{ha}^{-1} \mathrm{ano}^{-1}$, valores médios de IAF de 0,98 e 1,76 e sobrevivência de 7,3\% e 24,9\% para lotação contínua e rotacionada, respectivamente. Crioula e CUF-101 são as mais estacionais sob ambos os métodos. ABT-805 tem a maior produtividade sob pastejo nas condições edafoclimáticas da região.

Termos para indexação: Medicago sativa, produtividade, acúmulo de forragem, persistência.

\section{Seasonal yield, leaf area index and light interception of alfalfa cultivars under grazing}

\begin{abstract}
Grazed alfalfa (Medicago sativa L.) may reduce feeding costs of ruminant livestock but the agronomic performance of cultivars under grazing in Brazil is little known. The objective of this work was to evaluate the effect of the grazing method on herbage accumulation (HA), leaf area index (LAI) and light interception (LI) of five alfalfa cultivars over 295 days in Piracicaba, SP, Brazil. The trial was set up in a randomized complete block design and a split-plot arrangement (methods in the whole-plots and cultivars in the subplots). ABT-805 was the highest yielding entry under both continuous $\left(26,600 \mathrm{~kg} \mathrm{ha}^{-1} \mathrm{year}^{-1}\right)$ and rotational $\left(18,000 \mathrm{~kg} \mathrm{ha}^{-1}\right.$ year $\left.{ }^{-1}\right)$ stocking. It also had the highest mean LAI (2.1) and LI (53\%) under rotational stocking. Alfagraze, one of the lowest yielding cultivars under rotational stocking $\left(13,300 \mathrm{~kg}^{-1}\right.$ ha year $\left.{ }^{-1}\right)$ sustained similar total forage accumulation to that of ABT-805 under continuous stocking $\left(26,300 \mathrm{~kg} \mathrm{ha}^{-1}\right.$ year- $\left.^{-1}\right)$, with a mean LI of $47 \%$ and mean LAI between 1.1 and 1.8 for continuous and rotational stocking, respectively. Pioneer 5432 had a mean LI of $49 \%$, total seasonal herbage accumulation of 24,300 and 15,300 kg ha-1 year-1, mean LAI of 0.98 and 1.76, and mean survival of 7.3 and $24.9 \%$ under continuous and rotational stocking, respectively. Crioula and CUF-101 show a more pronounced seasonal growth under both grazing methods. ABT-805 is the best entry under grazing and under local conditions.
\end{abstract}

Index terms: Medicago sativa, productivity, herbage accumulation, persistence.

\section{Introdução}

No Brasil, a criação de ruminantes, responsáveis pela maior parte da produção de carne e leite, baseia-se na utilização de pastagens, por ser a forma mais econômica de fornecer alimento aos animais. No entanto, os índices de produtividade animal nas pastagens brasi- leiras ainda são baixos, em consequiência da qualidade das espécies forrageiras utilizadas, do baixo potencial produtivo do rebanho, das áreas de pastagens degradadas, do nível tecnológico das explorações e do pouco rendimento da atividade. Isso reduz a capacidade de investimento, por parte dos produtores, na modernização da pecuária (Pereira, 2002). 
A alfafa (Medicago sativa L.) é atualmente cultivada em aproximadamente 30.000 hectares no Brasil (Basigalup, 2000), com produtividade média anual em torno de 15.000 a $20.000 \mathrm{~kg} \mathrm{ha}^{-1}$ (Botrel \& Alvim, 1997; Oliveira et al., 2001). As maiores dificuldades para a expansão do uso da alfafa no Brasil estão associadas à falta de conhecimento sobre seu manejo em variadas formas de utilização, falta de cultivares adaptadas às diferentes regiões do país, baixa fertilidade do solo e manejo inadequado (Paim, 1994), e à escassez de cultivares adequadas ao cultivo em condições tropicais (Lédo et al., 2002). A cultivar Crioula, considerada o material nacional melhor adaptado, é a mais utilizada, embora existam outros materiais que vêm sendo testados pela RENACAL (Rede Nacional de Avaliação de Cultivares de Alfafa), com produtividades similares ou superiores, apesar de problemas de persistência (Dall'Agnol \& Scheffer-Basso, 2000). O programa de melhoramento da Embrapa Gado de Leite deu origem a uma população denominada Crioula CNPGL 1, com boa adaptação às condições edafoclimáticas da região da Zona da Mata de Minas Gerais, situando-se entre as cultivares mais produtivas (Botrel et al., 2002).

A produção de alfafa no país é destinada preferencialmente à fenação. A maioria das pesquisas relatadas foi conduzida com alfafa sob corte, sendo escassos os estudos sob pastejo. Oliveira et al. (2001) trabalharam com 12 cultivares de alfafa, durante dois anos (20 cortes sucessivos), realizando quatro pastejos com duração de 1 a 2 dias. Algumas cultivares - Crioula Chilena, UC-Cibola, Mecca, Mecca II e Pioneer 5312 - mantiveram a produtividade, entre as quais Crioula Chilena sem redução no número de coroas, e Pionner 5312 sem diminuição do número de hastes. Outras - Crioula Brasileira, Victoria, Moapa 69, WL 516, WL 605 e Pioneer XA132 - apresentaram decréscimo de produtividade no decorrer do ensaio.

Entre as cultivares disponíveis no mercado mundial, a Alfagraze foi desenvolvida para alta persistência sob pastejo (Bouton et al., 1991), possuindo boa produtividade de forragem e de sementes (Smith \& Bouton, 1993), mas com desempenho produtivo nas condições tropicais ainda pouco conhecido. Existe necessidade de pesquisa, tanto de material local como das introduções de alfafa importada, e também da capacidade produtiva e da adaptação às condições edafoclimáticas do Brasil, sob pastejo. Características morfológicas como área foliar e ambiente luminoso podem ser úteis para a compreensão das relações causa:efeito.

Pesq. agropec. bras., Brasília, v.39, n.10, p.1041-1048, out. 2004
O objetivo deste trabalho foi avaliar o efeito do método de pastejo sobre o acúmulo de forragem, índice de área foliar e interceptação luminosa de cinco cultivares de alfafa, durante 295 dias de pastejo em Piracicaba, SP.

\section{Material e Métodos}

O experimento foi conduzido em área do Dep. de Zootecnia da Escola Superior de Agricultura Luiz de Queiroz, Universidade de São Paulo, em Piracicaba, SP (224' $30^{\prime \prime} \mathrm{S}, 4^{\circ} 38^{\prime} 0^{\prime \prime} \mathrm{W} ; 546$ m de altitude). O período experimental foi de 31 de março de 2001 a 19 de janeiro de 2002, com duração de 295 dias. O experimento seguiu um delineamento em blocos completos casualizados, com parcelas subdivididas, dez tratamentos e seis repetições. Nas parcelas foi alocado o método de pastejo lotação contínua, mimetizada mediante pastejos semanais, e lotação rotacionada, com pastejos a cada quatro semanas na primavera-verão e a cada seis semanas no outono-inverno. Em cada parcela havia cinco sub-parcelas de 2,1 $7,0 \mathrm{~m}$, nas quais foram alocadas as cultivares, escolhidas de modo a representar uma gama de combinações entre três fatores: grau de dormência, procedência e tolerância ao pastejo, os quais afetam o desempenho agronômico dessa espécie. Embora a adaptação às condições climáticas locais não tenha sido previamente avaliada, admitiu-se que era variável entre cultivares, com Crioula sendo o único material amplamente utilizado nas condições brasileiras e, portanto, o mais adaptado ao local do experimento.

O solo da área experimental é classificado como Nitossolo Vermelho eutroférrico típico (Embrapa, 1999). As amostras de solo coletadas nas profundidades de 0-20 e 20-40 cm revelaram, respectivamente, as seguintes características químicas: $\mathrm{pH}$ em $\mathrm{CaCl}_{2}, 6,3 \mathrm{e}$ 6,$2 ; 40$ e $29 \mathrm{~g} \mathrm{dm}^{-3}$ de $\mathrm{MO} ; 88$ e $64 \mathrm{mg} \mathrm{dm}^{-3}$ de $\mathrm{P}_{\text {resina }}$; 9,6 e $8,1 \mathrm{mmol}_{\mathrm{c}} \mathrm{dm}^{-3}$ de $\mathrm{K} ; 103$ e $88 \mathrm{mmol}_{\mathrm{c}} \mathrm{dm}^{-3} \mathrm{de} \mathrm{Ca}$; 34 e $29 \mathrm{mmol}_{\mathrm{c}} \mathrm{dm}^{-3} \mathrm{de} \mathrm{Mg} ; 20$ e $22 \mathrm{mmol}_{\mathrm{c}} \mathrm{dm}^{-3} \mathrm{de} \mathrm{H}+\mathrm{Al}$. A área foi irrigada por aspersão, sempre que necessário, tomando-se como base a leitura de dois tensiômetros de cápsula porosa e em coluna de mercúrio, calibrados para $60 \%$ de umidade do solo, resultando em sessões de 15 minutos de rega, em média (lâmina d'água de $5 \mathrm{~mm}$ ).

As sementes foram submetidas à inoculação de BIOMAX contendo turfa esterilizada e a estirpe de Sinorhizobium meliloti SEMIA-134. A semeadura foi realizada em 1 o de setembro de 2000 , com espaçamento entrelinhas de $20 \mathrm{~cm}$, na densidade de $12 \mathrm{~kg}$ de semen- 
tes ha ${ }^{-1}$. O controle das ervas daninhas foi feito com glyphosate [N-(fosfonometil) glicina] pulverizado a 3\% $\mathrm{v} / \mathrm{v}$, e corte com roçadeira costal ao redor das parcelas. Em 19 de setembro de 2000 foi feita aplicação do graminicida haloxyfop-methyl [metil 2-\{4 (3-cloro-5(trifluorometil-2-piridinoxi)) fenoxi\}-propanoato], 0,09 a $0,12 \mathrm{~kg} \mathrm{ha}^{-1}$ do i.a., juntamente com óleo mineral Joint a $0,25 \%$.

Em 28 de novembro de 2000 foi realizado um corte de uniformização com segadora usada em fenação. Em 6 de dezembro de 2000 aplicaram-se $40 \mathrm{~kg} \mathrm{ha}^{-1}$ de FTE BR-12 para fornecimento de micronutrientes. Em fevereiro de 2001, foram montadas as cercas elétricas ao redor das parcelas para o pastejo.

Em 23 de fevereiro de 2001, realizou-se outro corte na área experimental, à altura média de $5 \mathrm{~cm}$ e, em 20 de março de 2001, foi feita outra aplicação do graminicida com óleo mineral. Em 17 de julho de 2001, em virtude da proliferação de pulgão-verde-azulado (Acyrthosiphon kondoi), aplicou-se Boveril PM [Beauveria bassiana (Bals.) Viull.] como controle biológico, na dose de $4 \mathrm{~kg} \mathrm{ha}^{-1}$.

O primeiro pastejo foi realizado em 31 de março de 2001, usando novilhas da raça Holandês preto e branco, com 12 a 18 meses de idade e peso vivo entre 300 e $450 \mathrm{~kg}$. Durante o período experimental foram realizados 43 e 10 pastejos nas parcelas sob o tratamento lotação contínua e lotação rotacionada, respectivamente. $\mathrm{O}$ pastejo era encerrado sempre que a altura média do dossel atingia $7 \mathrm{~cm}$.

A massa de forragem (MF) foi medida imediatamente antes e depois de cada pastejo, em cada unidade experimental. Utilizou-se a amostragem dupla, em que o método indireto foi o prato ascendente (Santillan et al., 1979), calibrado no pré e pós-pastejo (Pedreira, 2002) em quatro ocasiões. A calibração consistiu na demarcação, com um anel metálico que incluía uma área de 0,25 $\mathrm{m}^{2}$, as áreas de máxima e mínima MF em cada subparcela. Nessas áreas foram feitas leituras com o prato ascendente e, em seguida, a forragem contida no interior do anel foi cortada a $3 \mathrm{~cm}$ da superfície do solo. As amostras foram secadas em estufa de ar forçado a $65^{\circ} \mathrm{C}$ até peso constante e pesadas. A calibração do prato foi feita em 24 de março, 24 de maio, 27 de outubro e 20 de dezembro de 2001, num total de 120 estações de dupla amostragem. Com o uso de análise de regressão, foi estabelecida a relação entre a altura do prato e a MF. Em cada amostragem, a MF em cada subparcela era estimada indiretamente pela média de 30 leituras de prato. O acúmulo de forragem (AF) de cada ciclo foi calculado subtraindo-se a MF pós-pastejo do ciclo "n-1" da MF pré-pastejo do ciclo "n". As taxas médias diárias de AF (TMDAF) foram calculadas dividindo-se o AF pelo número de dias de acúmulo em cada rebrotação.

$\mathrm{O}$ índice de área foliar (IAF) foi medido com um analisador de dossel LI-COR modelo LAI 2000 (LICOR, Lincoln Nebraska, EUA), imediatamente antes e depois do pastejo, nas primeiras horas do dia ou no final da tarde em 31 de março de 2001, 24 de maio de 2001, 29 de setembro de 2001 e 18 de janeiro de 2002, ou seja, 0, 54, 182, e 293 dias após o início do pastejo, respectivamente. Em cada data de amostragem, foram realizadas leituras em dez pontos representativos, cinco nas linhas e cinco nas entrelinhas, da condição média da vegetação de cada subparcela. Na análise estatística foram utilizados valores médios [( $\left.\left.\mathrm{IAF}_{\text {pré }}+\mathrm{IAF}_{\mathrm{pós}}\right) / 2\right]$ para que a condição média da rebrotação em cada dossel fosse representada.

A interceptação luminosa (IL) foi estimada a partir de medições feitas com um fotômetro de barra de $1 \mathrm{~m}$ de comprimento, acoplado a um medidor LI-250 (LICOR, Lincoln Nebraska, EUA), antes e depois do pastejo, em 26 de maio e 24 de outubro de 2001, em oito pontos representativos da condição média da vegetação de cada parcela, sendo quatro leituras acima e quatro na base do dossel. O aparelho era posicionado obliquamente, com um extremo do sensor numa linha e outro extremo na outra linha do alfafal. As leituras foram feitas ao redor das $12 \mathrm{~h}$ sob céu claro, medindo-se a intensidade luminosa acima do alfafal $\left(\mathrm{I}_{\mathrm{o}}\right)$ e no nível do solo (I). A interceptação luminosa foi calculada como $\mathrm{IL}=\left[\left(\mathrm{I}_{0}-\mathrm{I}\right) / \mathrm{I}_{\mathrm{o}}\right] \times 100$. Foram utilizados valores médios na análise estatística [( $\left.\left.\mathrm{IL}_{\text {pré }}+\mathrm{IL}_{\mathrm{pós}}\right) / 2\right]$ com a finalidade de representar a condição média dos dosséis em cada rebrotação.

Os dados foram analisados utilizando-se o procedimento MIXED do pacote estatístico SAS (Littell et al., 1996). Em todos os casos, a cultivar, o método de pastejo e as interações entre ambos foram considerados efeitos fixos. Repetição e estação foram considerados efeitos aleatórios. No cálculo das médias foi utilizado o método dos quadrados mínimos (LSMEANS), e as comparações entre elas foram realizadas pelo teste de Tukey a $5 \%$ de probabilidade. As interações significativas foram desdobradas de acordo com os fatores envolvidos. 


\section{Resultados e Discussão}

\section{Acúmulo total, estacional, e taxas de acúmulo de forragem}

Os valores médios de AF variaram entre 26.600 (ABT805) e $19.400 \mathrm{~kg} \mathrm{ha}^{-1}$ ano $^{-1}$ (CUF-101) sob lotação contínua, e entre 18.300 (Crioula) e $13.300 \mathrm{~kg} \mathrm{ha}^{-1} \mathrm{ano}^{-1}$ (Alfagraze) sob lotação rotacionada (Tabela 1). Os AF sob lotação contínua correspondentes às cultivares Crioula (19.900 kg ha ${ }^{-1}$ ano $\left.^{-1}\right)$ e CUF-101 (19.400 kg ha-1 ano $\left.^{-1}\right)$ não diferiram entre si, embora tenham divergindo daqueles das cultivares tolerantes ao pastejo. Os maiores AF medidos sob lotação rotacionada foram para Crioula (18.300 kg ha-1 ano $\left.{ }^{-1}\right)$, ABT-805 (17.600 kg ha ${ }^{-1}$ ano $\left.^{-1}\right) \mathrm{e}$ CUF-101 (17.700 kg ha-1 ano $\left.^{-1}\right)$. Pioneer $5432 \mathrm{e} \mathrm{Alfagraze}^{-1}$ tiveram produtividades semelhantes (entre $15.300 \mathrm{e}$ $13.300 \mathrm{~kg} \mathrm{ha}^{-1} \mathrm{ano}^{-1}$ ) (Tabela 1).

Resultados deste estudo corroboram os oriundos de pesquisas conduzidas com esses materiais, sob corte. Oliveira et al. (2001) obtiveram, após dois anos de corte, produtividade média em torno de 21.000 (Crioula

Tabela 1. Acúmulo de forragem total (AFT), acúmulo de forragem de verão (AFV) e de inverno (AFI) de cultivares de alfafa sob pastejo, ao longo do período experimental ${ }^{(1)}$.

\begin{tabular}{|c|c|c|}
\hline \multirow[t]{2}{*}{ Cultivar } & \multicolumn{2}{|c|}{ Método de pastejo } \\
\hline & Lotação contínua & Lotação rotacionada \\
\hline & \multicolumn{2}{|c|}{ AFT $\left(\mathrm{kg} \mathrm{ha}^{-1} \mathrm{ano}^{-1}\right)$} \\
\hline ABT-805 & $26.600 \mathrm{Aa}$ & $17.600 \mathrm{Ab}$ \\
\hline Alfagraze & $26.300 \mathrm{Aa}$ & $13.300 \mathrm{Bb}$ \\
\hline Crioula & $19.900 \mathrm{Bb}$ & $18.300 \mathrm{Ab}$ \\
\hline CUF-101 & $19.400 \mathrm{Bb}$ & $17.700 \mathrm{Ab}$ \\
\hline \multirow[t]{2}{*}{ Pioneer 5432} & $24.300 \mathrm{Aa}$ & $15.300 \mathrm{Bb}$ \\
\hline & \multicolumn{2}{|c|}{$\operatorname{AFV}\left(\mathrm{kg} \mathrm{ha}^{-1} \mathrm{ano}^{-1}\right)$} \\
\hline ABT-805 & $18.900 \mathrm{Aa}(71,0 \mathrm{~A})$ & $14.300 \mathrm{Ab}(81,2 \mathrm{~A})$ \\
\hline Alfagraze & $18.800 \mathrm{Aa}(71,5 \mathrm{~A})$ & $10.600 \mathrm{Cb}(79,7 \mathrm{C})$ \\
\hline Crioula & $12.800 \mathrm{Ca}(64,3 \mathrm{C})$ & $14.700 \mathrm{Aa}(80,3 \mathrm{~B})$ \\
\hline CUF-101 & $12.200 \mathrm{Ca}(62,9 \mathrm{C})$ & $14.300 \mathrm{Aa}(80,8 \mathrm{~B})$ \\
\hline \multirow[t]{2}{*}{ Pioneer 5432} & $16.600 \mathrm{Ba}(68,3 \mathrm{~B})$ & $12.600 \mathrm{Bb}(82,3 \mathrm{~A})$ \\
\hline & \multicolumn{2}{|c|}{ AFI $\left(\mathrm{kg} \mathrm{ha}^{-1} \mathrm{ano}^{-1}\right)$} \\
\hline ABT-805 & $7.600 \mathrm{Aa}(28,6 \mathrm{C})$ & $3.300 \mathrm{Ab}(18,7 \mathrm{C})$ \\
\hline Alfagraze & $7.400 \mathrm{Aa}(28,1 \mathrm{C})$ & $2.700 \mathrm{Bb}(20,3 \mathrm{~A})$ \\
\hline Crioula & $7.200 \mathrm{Aa}(36,0 \mathrm{~A})$ & $3.600 \mathrm{Ab}(19,7 \mathrm{~B})$ \\
\hline CUF-101 & $7.300 \mathrm{Aa}(37,5 \mathrm{~A})$ & $3.400 \mathrm{Ab}(19,2 \mathrm{~B})$ \\
\hline Pioneer 5432 & $7.700 \mathrm{Aa}(31,8 \mathrm{~B})$ & $3.200 \mathrm{Ab}(20,9 \mathrm{~A})$ \\
\hline
\end{tabular}

${ }^{(1)}$ Médias seguidas de letras iguais, minúsculas nas linhas e maiúsculas nas colunas, não diferem entre si pelo teste de Tukey a 5\% de probabilidade; valores entre parênteses indicam porcentagem de AFV e AFI em relação ao AFT; esses valores, seguidos da mesma letra maiúscula na coluna não diferem entre si pelo teste de Tukey a $5 \%$ de probabilidade, dentro de AFT, dentro de AFV e dentro de AFI; $\mathrm{EPM}_{\mathrm{AFT}}=939,3 \mathrm{~kg} \mathrm{ha}^{-1} \mathrm{ano}^{-1}$; $\mathrm{EPM}_{\mathrm{AFV}}=852,2 \mathrm{~kg} \mathrm{ha}^{-1} \mathrm{ano}^{-1} ; \mathrm{EPM}_{\mathrm{AFI}}=288,2 \mathrm{~kg} \mathrm{ha}^{-1} \mathrm{ano}^{-1}$.
Brasileira) e $25.000 \mathrm{~kg} \mathrm{ha}^{-1} \mathrm{ano}^{-1}$ (Crioula Chilena). Produtividades semelhantes foram alcançadas em Crioula importada, Crioula CRA-RS e Crioula Honda (Ruggieri et al., 2002).

Smith et al. (1989) avaliaram a GA-GC (atualmente Alfagraze), identificando o seu potencial quanto ao vigor de rebrotação e persistência sob pastejo, com produtividade de $6.370 \mathrm{~kg} \mathrm{ha}^{-1}$ durante 114 dias de pastejo no verão. Smith \& Bouton (1993) mediram em Alfagraze produtividades em torno de 14.000 a $17.000 \mathrm{~kg} \mathrm{ha}^{-1} \mathrm{ano}^{-1}$ sob corte para feno e de $7.505 \mathrm{~kg} \mathrm{ha}^{-1} \mathrm{ano}^{-1} \mathrm{em}$ dois anos de pastejo, sob lotação contínua. Neste estudo, o desempenho produtivo da Alfagraze foi de 26.300 e $13.300 \mathrm{~kg} \mathrm{ha}^{-1} \mathrm{ano}^{-1}$ sob lotação contínua e rotacionada, respectivamente.

Dos 295 dias de período experimental, 70,2\% foram considerados verão e $29,8 \%$ inverno, usando um critério de conveniência, de modo a incluir dois ciclos de pastejo completos (cerca de 12 semanas) no inverno. $\mathrm{O}$ acúmulo de forragem de verão (AFV) sob lotação contínua foi maior em ABT-805 e Alfagraze, intermediário em Pioneer 5432, e menor em Crioula e CUF-101. Sob lotação rotacionada, os AFV foram maiores em Crioula, CUF-101 e ABT-805, intermediário em Pioneer 5432 e menor em Alfagraze. A produtividade de verão da Pioneer 5432 foi intermediária em ambos os métodos de pastejo (Tabela 1).

Um contraste entre as cultivares tolerantes e não tolerantes ao pastejo ficou evidente, sendo mais pronunciado sob lotação contínua que sob lotação rotacionada. Com relação ao AFV sob lotação contínua, as cultivares ABT-805 e Alfagraze concentraram cerca de $71 \%$ da sua produção total no verão, enquanto Pioneer 5432, aproximadamente 68\%, Crioula e CUF-101, 63,6\%, com produções em torno de $19.000,17.000 \mathrm{e}$ $13.000 \mathrm{~kg} \mathrm{ha}^{-1} \mathrm{ano}^{-1}$, respectivamente (Tabela 1). Sob lotação rotacionada, os AFV foram distribuídos em três grupos: o primeiro, composto por Pioneer 5432 e ABT-805, em que cerca de $82 \%$ do AF total correspondeu a AFV; o segundo, composto por Crioula e CUF-101, cuja proporção foi de $80,5 \%$; e um terceiro grupo distinto para Alfagraze, que produziu 79,7\% do total anual durante o verão. As produtividades estiveram em torno de 13.000, 14.500 e $11.000 \mathrm{~kg} \mathrm{ha}^{-1} \mathrm{ano}^{-1}$ respectivamente, para os três grupos. Ruggieri et al. (2002) obtiveram, no verão, produtividades de $8.210 \mathrm{e} 8.000 \mathrm{~kg} \mathrm{ha}^{-1} \mathrm{ano}^{-1}$ para Crioula importada e Crioula CRA-RS, respectivamente. 
Sob lotação rotacionada, os acúmulos de forragem de inverno (AFI) da Crioula, CUF-101, ABT-805 e Pioneer 5432, foram semelhantes, todos superiores ao da Alfagraze (2.700 kg ha-1 ano $\left.{ }^{-1}\right)$, que apresentou a menor produtividade (Tabela 1). O AFI entre as cultivares não diferiu sob lotação contínua, com produtividade média em torno de $7.400 \mathrm{~kg} \mathrm{ha}^{-1} \mathrm{ano}^{-1}$, o que correspondeu a cerca de $37 \%$ do total anual em Crioula e CUF-101, 28\% em ABT-805 e Alfagraze e $32 \%$ em Pioneer 5432. Sob lotação rotacionada, as produções de inverno ficaram em torno de $3.500 \mathrm{~kg} \mathrm{ha}^{-1} \mathrm{ano}^{-1}$ para ABT-805, Crioula, CUF-101 e Pioneer 5432, e $2.700 \mathrm{~kg} \mathrm{ha}^{-1}$ para Alfagraze, correspondendo a $19,6 \%$ (média das quatro primeiras) e $20,3 \%$ (Alfagraze) da produção total do ano (Tabela 1). Ruggieri et al. (2002) verificaram produtividade de inverno de $2.420 \mathrm{~kg} \mathrm{ha}^{-1} \mathrm{ano}^{-1}$ (Crioula importada) e $2.440 \mathrm{~kg} \mathrm{ha}^{-1} \mathrm{ano}^{-1}$ (Crioula CRA-RS), acontecendo o mesmo com os acúmulos de verão descritos e discutidos anteriormente. Botrel \& Alvim (1997) obtiveram, na Zona da Mata de Minas Gerais, média de $47 \%$ da produção anual no inverno, ao passo que Oliveira (2001) observou média de $41,2 \%$ do total anual produzido nos meses de inverno em Piracicaba, SP.

A taxa média diária de acúmulo de forragem no verão (TMDAV) foi maior sob lotação contínua, para ABT-805 e Alfagraze, intermediária para Pioneer 5432 e menor para Crioula e CUF-101. Sob lotação rotacionada, as TMDAV foram maiores em Crioula, ABT-805 e CUF-101, enquanto Pioneer 5432 e Alfagraze apresentaram as menores taxas (Tabela 2). Os valores médios de taxa média diária de acúmulo de forragem no inverno (TMDAI) sob lotação contínua não diferiram entre Pioneer 5432, ABT-805 e Alfagraze, mas divergiram dos de Crioula e CUF-101. Sob lotação rotacionada, os valores médios das TMDAI para as cultivares Crioula, CUF-101, ABT-805 e Pioneer 5432 foram superiores ao da Alfagraze, que apresentou a menor taxa de acúmulo de inverno (Tabela 2). Os acúmulos e as taxas médias diárias de acúmulo de forragem foram sempre superiores sob lotação contínua do que sob lotação rotacionada, exceto em Crioula e CUF-101. Alfagraze, apesar de ter sido uma das duas cultivares menos produtivas sob lotação rotacionada, foi uma das três mais produtivas sob lotação contínua.

Sob lotação contínua, as TMDAV foram apenas $30 \%$ superiores às TMDAI. Isto pode estar relacionado com o inverno mais quente que a média histórica na localidade do experimento. Monteiro et al. (1999), trabalhando em Piracicaba com as cultivares CUF-101 e Crioula, relataram que, quando as temperaturas médias do in- verno se mantiveram mais baixas dentro do período experimental, as plantas responderam de maneira semelhante ao manejo da desfolha, enquanto após o estabelecimento de temperaturas mais altas, com média de $24^{\circ} \mathrm{C}$, registraram-se diferenças quanto ao vigor de rebrotação e a produção de forragem. Os mesmos materiais, sob pastejo, apresentaram desempenho semelhante, com AF em torno de $20.000 \mathrm{~kg} \mathrm{ha}^{-1} \mathrm{ano}^{-1} \mathrm{sob}$ lotação contínua e de $18.000 \mathrm{~kg} \mathrm{ha}^{-1} \mathrm{ano}^{-1}$ sob lotação rotacionada.

A dormência e a propriedade de sobreviver a invernos rigorosos são características importantes da alfafa, mas nem as bases fisiológicas desses mecanismos nem a interação entre fisiologia e ambiente estão ainda completamente esclarecidas (Cunningham et al., 2001). O uso de cultivares não dormentes pode ser desejável em condições tropicais, principalmente sob irrigação, pois estas produzem maiores quantidades de forragem durante o outono e inverno, reassumindo o crescimento das hastes no início da primavera e iniciando a rebrotação após um corte no verão.

\section{Índice de área foliar}

Os valores de IAF variaram entre 2,07 (ABT-805) e 1,76 (Pioneer 5432) sob lotação rotacionada e entre 1,05 (Alfagraze) e 0,91 (ABT-805), sob lotação contínua (Tabela 3). O IAF médio sob lotação contínua decresceu com o avanço da estação de pastejo, variando entre 1,64, 0,83, 0,74 e 0,22 no início, aos 54, 182 e 293 dias

Tabela 2. Taxa média diária de acúmulo de forragem no verão (TMDAV) e no inverno (TMDAI) de cultivares de alfafa sob pastejo, durante os 191 e 81 dias correspondentes aos períodos de verão e inverno, respectivamente ${ }^{(1)}$.

\begin{tabular}{lcc}
\hline Cultivar & \multicolumn{2}{c}{ Método de pastejo } \\
\cline { 2 - 3 } & \multicolumn{2}{c}{ TMDAV $\left(\mathrm{kg} \mathrm{ha}^{-1} \mathrm{dia}^{-1}\right)$} \\
ABT-805 & 139,09Aa & $92,07 \mathrm{Ab}$ \\
Alfagraze & $137,60 \mathrm{Aa}$ & $69,61 \mathrm{Cb}$ \\
Crioula & $104,46 \mathrm{Ca}$ & $95,99 \mathrm{Aa}$ \\
CUF-101 & $101,59 \mathrm{Ca}$ & $92,80 \mathrm{Aa}$ \\
Pioneer 5432 & $127,51 \mathrm{Ba}$ & $80,19 \mathrm{Bb}$ \\
\hline & \multicolumn{2}{c}{ TMDAI $\left(\mathrm{kg} \mathrm{ha}^{-1} \mathrm{dia}^{-1}\right)$} \\
ABT-805 & $93,82 \mathrm{Aa}$ & $40,98 \mathrm{Ab}$ \\
Alfagraze & $91,21 \mathrm{Aa}$ & $33,64 \mathrm{Bb}$ \\
Crioula & $88,73 \mathrm{Ba}$ & $44,92 \mathrm{Ab}$ \\
CUF-101 & $89,86 \mathrm{Ba}$ & $42,15 \mathrm{Ab}$ \\
Pioneer 5432 & $95,60 \mathrm{Aa}$ & $40,11 \mathrm{Ab}$ \\
\hline
\end{tabular}

(1)Médias seguidas por letras iguais, minúsculas nas linhas e maiúsculas nas colunas, não diferem entre si pelo teste de Tukey a $5 \%$ de probabilidade; $\mathrm{EPM}_{\mathrm{TMDAV}}=4,87 \mathrm{~kg} \mathrm{ha}^{-1} \mathrm{dia}^{-1}, \mathrm{EPM}_{\mathrm{TMDAI}}=3,70 \mathrm{~kg} \mathrm{ha}^{-1} \mathrm{dia}^{-1}$. 
após o início do pastejo, respectivamente (Figura 1). Essa redução evidencia tendência à degradação dos estandes sob esse tipo de utilização. Sob lotação rotacionada, os valores de IAF variaram entre 1,96 no início e 1,08 aos 293 de pastejo. Houve um aumento nos valores de IAF aos 54 e 182 dias após o início do pastejo, correspondendo a 2,10 e 2,41 , respectivamente.

A redução do IAF sob lotação contínua provavelmente ocorreu por causa da frequência semanal de desfolha - usada para mimetizar essa modalidade de manejo - e da alta intensidade de desfolha, o que impediu os estandes de reporem rapidamente a área foliar. $\mathrm{O}$ aumento do IAF médio aos 54 e 182 dias depois de iniciado o pastejo no método rotacionado pode ter ocorrido por causa das temperaturas atipicamente altas durante o inverno, favorecendo o aparecimento e a expansão foliar.

Alfagraze, Pioneer 5432 e ABT-805 são cultivares tolerantes ao pastejo e isso pode estar associado aos maiores valores de IAF no método contínuo, em comparação com os IAF médios de Crioula e de CUF-101, sugerindo a menor tolerância ao pastejo destas últimas (Tabela 3). Em estudo paralelo, as taxas de sobrevivência de ABT-805, Alfagraze e Pioneer 5432, sob lotação contínua, foram de $10,7 \%, 25,9 \%$ e $7,3 \%$, respectivamente. Crioula e CUF-101 não sobreviveram (0\%) ao pastejo sob lotação contínua até o final do experimento.

Tabela 3. Índice de área foliar médio $\left[\left(\mathrm{IAF}_{\text {pré }}+\mathrm{IAF}_{\mathrm{pós}}\right) / 2\right] \mathrm{e}$ interceptação luminosa média (IL) em dosséis de cultivares de alfafa sob pastejo, ao longo do período experimental ${ }^{(1)}$.

\begin{tabular}{|c|c|c|}
\hline \multirow[t]{2}{*}{ Cultivar } & \multicolumn{2}{|c|}{ Método de pastejo } \\
\hline & Lotação contínua & Lotação rotacionada \\
\hline & \multicolumn{2}{|c|}{ IAF } \\
\hline ABT-805 & $0,91 \mathrm{Aa}$ & $2,07 \mathrm{Ab}$ \\
\hline Alfagraze & $1,05 \mathrm{Aa}$ & $1,80 \mathrm{Bb}$ \\
\hline Crioula & n.d..$^{(2)}$ & $1,96 \mathrm{~A}$ \\
\hline CUF-101 & n.d. & $1,83 \mathrm{~A}$ \\
\hline \multirow[t]{2}{*}{ Pioneer 5432} & $0,98 \mathrm{Aa}$ & $1,76 \mathrm{Bb}$ \\
\hline & \multicolumn{2}{|c|}{$\operatorname{IL}(\%)$} \\
\hline ABT-805 & $13,44 \mathrm{Ab}$ & $52,66 \mathrm{Aa}$ \\
\hline Alfagraze & $14,10 \mathrm{Ab}$ & $47,15 \mathrm{Ba}$ \\
\hline Crioula & $10,72 \mathrm{Bb}$ & $51,96 \mathrm{Aa}$ \\
\hline CUF-101 & $10,88 \mathrm{Bb}$ & $49,29 \mathrm{Ba}$ \\
\hline Pioneer 5432 & $12,56 \mathrm{Ab}$ & $48,94 \mathrm{Ba}$ \\
\hline
\end{tabular}

(1)Médias seguidas de letras iguais, minúsculas nas linhas e maiúsculas nas colunas, não diferem entre si pelo teste de Tukey a 5\% de probabilidade; o erro-padrão da média em relação a IAF e a IL foi de 0,054 e 1,177, respectivamente. ${ }^{(2)}$ Dados não disponíveis devido à degradação do estande de plantas.
Elevado vigor de rebrotação e acúmulo de forragem estão associados a maiores valores de IAF e à forma como a planta retoma o crescimento após o corte ou pastejo. Os maiores AF e menores IAF foram obtidos sob lotação contínua (Tabelas 1 e 3), embora o bom desempenho das cultivares sob lotação rotacionada possa ser explicado pela capacidade das plantas de repor área foliar suficiente, mantendo boas coberturas do solo e bons estandes após 295 dias de pastejo.

O desempenho das cultivares tolerantes ao pastejo (ABT-805, Alfagraze e Pioneer 5432) sob lotação contínua está provavelmente relacionado com a melhor resposta dessas plantas ao estresse da desfolha e pisoteio pelos animais. No entanto, a cultivar Crioula, apesar de supostamente adaptada às condições locais, não se destacou, possivelmente pela falta de tolerância ao pastejo. Basigalup (2000) afirma que alfafais sob lotação contínua sofrem perda de vigor, produzindo menor quantidade de forragem e reduzindo o tamanho do sistema radicular.

Sob lotação rotacionada, o maior IAF médio foi de ABT-805, seguido por Crioula e CUF-101. A ABT-805, uma cultivar importada e tolerante ao pastejo, ficou entre as de melhor desempenho. Avice et al. (1997) registraram IAF de 4,7, 3,5 e 3,2 no primeiro, segundo e terceiro corte efetuados quando a alfafa estava com 8,10 e $14 \%$ de floração. Após o terceiro corte e com uma rebrotação de 21 dias, o IAF foi 2,9 , sugerindo que com esse valor a planta já tinha iniciado o acúmulo de reservas. Os mesmos autores constataram que com valores de

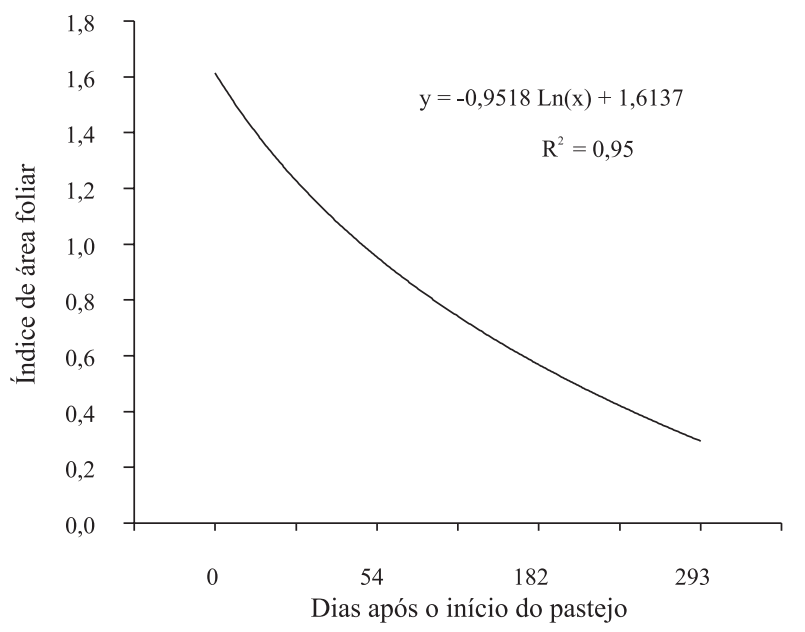

Figura 1. Variação do índice de área foliar (IAF) médio dos dosséis de alfafa sob lotação contínua, ao longo do período experimental. 
IAF superiores a 4, houve perdas de folhas no estrato inferior, até mesmo durante o primeiro e terceiro corte.

\section{Interceptação luminosa}

Os valores médios de IL variaram entre $52,7 \%$ (ABT-805) e 47,1\% (Alfagraze), sob lotação rotacionada e $14,1 \%$ (Alfagraze) e 10,7\% (Crioula), sob lotação contínua (Tabela 3). A IL média sob lotação contínua diminuiu com o decorrer da estação de pastejo em $48 \%$, enquanto no pastejo rotacionado a queda foi de $11 \%$. Houve redução na IL no decorrer dos dias, sendo registrados valores em torno de $35 \%$ e $28 \%$ aos 57 e 151 dias de iniciado o pastejo, respectivamente.

Sob lotação contínua, os materiais tolerantes ao pastejo mostraram maiores valores de IL, enquanto CUF-101 e Crioula apresentaram os menores valores. Esses resultados são condizentes com os baixos AF dessas cultivares, os quais estão associados com a baixa persistência desses materiais, manifestada nas baixas produtividades nos ciclos finais da estação de pastejo. Sob lotação rotacionada, a IL em ABT-805 e Crioula foi maior, ao passo que CUF-101, Pioneer 5432 e Alfagraze evidenciaram os menores valores (Tabela 3 ).

Gosse et al. (1988) afirmaram que a contribuição da rebrotação a partir de gemas axilares, para a interceptação da radiação e produção de forragem em alfafa foi limitada, sendo de aproximadamente $30 \%$, enquanto a rebrotação proveniente de gemas da coroa interceptou cerca de $60 \%$ a $76 \%$ da radiação incidente. No entanto, a rebrotação proveniente de gemas axilares próximas à coroa pode ter um papel importante por causa da sua rápida recuperação após o corte, principalmente no período em que o desenvolvimento da área foliar é o principal fator limitante ao crescimento (Monteiro et al., 1999). No presente estudo, sob lotação rotacionada, ABT-805 e Crioula tiveram maior interceptação luminosa, com valores médios de $52,7 \%$ e $51,9 \%$, respectivamente, provavelmente por causa da rápida reposição de área foliar fotossintetizante, corroborando resultados descritos na literatura.

\section{Conclusões}

1. ABT-805 é a cultivar de melhor desempenho quanto à produtividade, índice de área foliar e intercepação luminosa, sob lotação contínua e rotacionada.

2. Alfagraze é a cultivar mais produtiva sob lotação contínua, e a menos produtiva sob lotação rotacionada.

3. Pioneer 5432 é intermediária em relação à produtividade e tolerância ao pastejo, ao passo que Crioula e
CUF-101 são medianamente produtivas, manifestando as características das chamadas alfafas tipo feno.

4. ABT-805 tem a vantagem de ser não-dormente e tolerante ao pastejo, condições essas desejáveis nos sistemas brasileiros de produção animal.

\section{Agradecimentos}

Ao convênio Capes/SPUA e à Fundação de Estudos Agrários Luiz de Queiroz, pela concessão de bolsa a María del Carmen Ferragine; à Fapesp, pela concessão de bolsa a Lyssa Otani e Felipe Tonato.

\section{Referências}

AVICE, J.C.; LEMAIRE, G.; OURRY, A.; BOUCAUD, J. Effects of the previous shoot removal frequency on subsequent shoot regrowth in two Medicago sativa L. cultivars. Plant and Soil, v.188, p.189-198, 1997.

BASIGALUP, D.H. Present and future of alfalfa as a grazing crop in south America. In: NORTH AMERICAN ALFALFA IMPROVEMENT CONFERENCE., 37., Madison, Proceedings, 2000. Disponível em: <http://www.naaic.org> Acesso em: 11 Jul. 2003.

BOTREL, M. de A.; ALVIM, M.J. Avaliação de cultivares de alfafa na Zona da Mata de Minas Gerais. Pesquisa Agropecuária Brasileira, v.32, p.971-975, 1997.

BOTREL, M. de A.; CARNEIRO, H.; SALES, E.C.J.; ALVIM, M.J.; LÉDO, J.D.S.; PEREIRA, A.V.; XAVIER, D.F. Produção e qualidade da forragem de cultivares de alfafa avaliadas na zona da Mata de Minas Gerais. In: REUNIÃO DA SOCIEDADE BRASILEIRA DE ZOOTECNIA, 39., Recife, 2002. Anais. Recife: SBZ, 2002. CD-ROM.

BOUTON, J.H.; SMITH, S.R.; WOOD, D.T.; HOVELAND, C.S.; BRUMMER, E.C. Registration of 'Alfagraze' Alfalfa. Crop Science, v.31, p.479, 1991.

CUNNINGHAM, S.M.; GANA, J.A.; VOLENEC, J.J.; TEUBER, L.R. Winter hardiness, root physiology and gene expression in successive fall dormancy selections from 'Mesilla' and 'CUF 101' alfalfa. Crop Science, v.41, p.1091-1098, 2001.

DALL'AGNOL, M.; SCHEFFER-BASSO, S.M. Produção e utilização de alfafa. In: SIMPÓSIO SOBRE MANEJO DE PASTAGENS, 17., Piracicaba, 2000. Anais. Piracicaba: Fealq, 2000. p.265-295.

EMBRAPA. Centro Nacional de Pesquisa de Solos (Rio de Janeiro, RJ). Sistema brasileiro de classificação de solos. Brasília: Embrapa-SPI; Embrapa-CNPS, 1999. 412p.

GOSSE, G.; LEMAIRE, G.; CHARTIER, M.; BALFOURIER, F. Structure of a lucerne population (Medicago sativa L.) and dynamics of stem competition for light during regrowth. Journal of Applied Ecology, v.25, p.609-617, 1988. 
LÉDO, F.J.S.; FERREIRA, R.D.P.; BOTREL, M. de A.; PEREIRA, A.V.; COELHO, A.D.F. Introdução e avaliação de germoplasma de alfafa no ecossistema de Mata Atlântica de Minas Gerais. In: REUNIÃO DA SOCIEDADE BRASILEIRA DE ZOOTECNIA, 39., Recife, 2002. Anais. Recife: SBZ, 2002. CD-ROOM.

LITTELL, R.C.; MILLIKEN, G.A.; STROUP, W.W.; WOLFINGER, R.D. SAS system for mixed models. Cary: SAS Institute, 1996. $633 \mathrm{p}$.

MONTEIRO, A.L.G.; CORSI, M.; CARVALHO, D.D. Freqüência de corte e intensidade de desfolha em duas cultivares de alfafa (Medicago sativa L.). I. Peso, número, produção estacional e dinâmica de aparecimento das brotações basilares. Revista Brasileira de Zootecnia, v.28, p.446-452, 1999.

OLIVEIRA, W.S. Seleção de cultivares de alfafa (Medicago sativa L.) eficientes em produção e qualidade da biomassa. 2001. 118p. Tese (Doutorado) - Centro de Energia Nuclear na Agricultura, Universidade de São Paulo, Piracicaba.

OLIVEIRA, W.S.; OLIVEIRA, P.P.A.; CORSI, M.; BOUTON, J.H.; TSAI, S.M. Avaliação preliminar de cultivares de alfafa sob pastejo com alta lotação animal e ciclos de curta duração. In: REUNIÃO DA SOCIEDADE BRASILEIRA DE ZOOTECNIA, 38., Piracicaba, 2001. Anais. Piracicaba: SBZ, 2001. p.115-117.

PAIM, N.R. Utilização e melhoramento da alfafa. In: WORKSHOP SOBRE POTENCIAL FORRAGEIRO DA ALFAFA (Medicago sativa L.) NOS TRÓPICOS, Juiz de Fora, 1994. Anais. Juiz de Fora: Embrapa-CNPGL, 1994. p.141-158.

PEDREIRA, C.G.S. Avanços metodológicos na avaliação de pastagens. In: REUNIÃO DA SOCIEDADE BRASILEIRA DE ZOOTECNIA, 39., Recife, 2002. Anais. Recife: SBZ, 2002. p.100150.

PEREIRA, A.V. Avanços no melhoramento genético de gramíneas forrageiras tropicais. In: REUNIÃO DA SOCIEDADE BRASILEIRA DE ZOOTECNIA, 39., Recife, 2002. Anais. Recife: SBZ, 2002. p.19-41.

RUGGIERI, A.C.; ALMEIDA, A.R.P.; FIGUEIREDO, L.A.; BOTREL, M.A. Comportamento de 20 cultivares de alfafa com 3 anos de cultivo em Sertãozinho-SP. In: REUNIÃO DA SOCIEDADE BRASILEIRA DE ZOOTECNIA, 39., Recife, 2002. Anais. Recife: SBZ, 2002. CD-ROM.

SANTILLAN, R.A.; OCUMPAUGH, W.R.; MOTT, G.O. Estimating forage yield with a disk meter. Agronomy Journal, v.71, p.71-74, 1979.

SMITH, S.R.; BOUTON, J.H. Selection within alfalfa cultivars for persistence under continuous stocking. Crop Science, v.33, p.13211328, 1993.

SMITH, S.R.; BOUTON, J.H.; HOVELAND, C.S. Alfalfa persistence and regrowth potential under continuous grazing. Agronomy Journal, v.81, p.960-965, 1989.

Recebido em 4 de dezembro de 2003 e aprovado em 10 de agosto de 2004 